\title{
Nanoparticles Containing Gel Formulation for the Treatment of Psoriasis
}

\author{
Ramila Prajapati ${ }^{1, *}$, Jayvadan Patel $^{2}$, Anita Patel ${ }^{2}$ \\ 'Department of Pharmacy, Sankalchand Patel University, Visnagar, Gujarat, INDIA. \\ 2Department of Pharmaceutics, Nootan Pharmacy College, Faculty of Pharmacy, Sankalchand Patel University, Visnagar, Gujarat, INDIA.
}

\begin{abstract}
Background: Psoriasis is a widespread chronic disease affecting $1-3 \%$ of total population and in most cases, treated by topical application of corticosteroids. Though, the topical route is very demanding because of the physicochemical nature of diseased stratum corneum and so no, single treatment works for every patient. The oral route is evidence for harsh side effects because of systemic immunosuppression which can be avoided by topical route. Objectives: The objective of the present study is to design, develop, and evaluate nanoparticulate gel containing Cyclosporine A for the treatment of psoriasis. Methods: Cyclosporine loaded nanoparticles were formulated by the modified nanoprecipitation technique and characterized for particle size, encapsulation efficiency, morphological analysis, drugpolymer compatibility study, powder x-ray diffraction analysis. Optimized formulation of Cyclosporine nanoparticles further converted into the gel using Carbopol 934. Results: Cyclosporine loaded nanoparticles showed a spherical shape with smooth surface. In vitro antiproliferative activity proved that cell viability was not affected with blank nanoparticles, as there was no effect of concentration and types of surfactant on cell viability causing no cell death. The gel formulation hydrates the skin which helps
\end{abstract}

in the higher permeation of Cyclosporine loaded nanoparticulate gel. Conclusion: In vitro anti-psoriatic study suggested that the developed Cyclosporine nanoparticulate gel formulation exhibits improved dermal delivery of Cyclosporine and also nanoparticles had a superior effect on cell growth as compared to the free Cyclosporine. Dermal pharmacokinetic study established that the amount of Cyclosporine reaching the viable layer is slightly high for nanoparticulate gel as compared to free Cyclosporine. Thus, Cyclosporine loaded nanoparticulate gel formulation might be the potential delivery system for the treatment of psoriasis.

Key words: Cyclosporine A, Dermal pharmacokinetic study, In vitro antipsoriatic study, Nanoprecipitation technique, Topical gel.

\section{Correspondence}

Mrs. Ramila Prajapati

Research Scholar, Department of Pharmacy, Sankalchand Patel University, Visnagar-384315, Gujarat, INDIA.

Phone no: +919898025640

Email: ramiladj@gmail.com

DOI: 10.5530/ijpi.2021.1.14

\section{INTRODUCTION}

Psoriasis is a prevailing, chronic inflammatory most common skin disorder affecting just about $0.5 \%-1 \%$ of children as well as $2 \%-3 \%$ of the world's population. ${ }^{1}$ It is a bi-modally distributed skin disorder with one main age of onset at 20-30 years in addition to a later on lesser peak of onset at 50-60 years of age. ${ }^{2,3}$ This is a T-cell mediated autoimmune disorder that leads to keratinocyte hyper proliferation. ${ }^{4}$ Psoriasis confers significant physical and psychological distress and impairment usually resulting in a detrimental impact on patient quality of life., ${ }^{5,6}$ The exact cause of the origin of the disease is unknown, but it is considered as a multifactorial disorder associated with an overexpression of proinflammatory chemokines and cytokines produced by Th1 cells. ${ }^{7,8}$

Cyclosporine A (CsA) is a potent immunosuppressive agent and used for the treatment of moderate to severe psoriasis. ${ }^{9} \mathrm{CsA}$ is a polypeptide calcineurin inhibitor that acts by inhibiting calcineurin, as a result of inhibition it decreases the production of Interleukin-2, which is helpful in the activation of T-cell. ${ }^{10}$ The key side effects of CsA after systemic administration for the treatment of psoriasis are hypertension, cancer, and nephrotoxicity with reduced renal transplant function ${ }^{11,12}$ which may be reduced by topical administration of CsA. Furthermore, the most frequent type is mild psoriasis, which mainly requires the topical treatment. In spite of several advantages offered by topical route, only a few molecules are administered topically because of the formidable barrier nature of the stratum corneum. A major problem for topical use of CsA is insufficient percutaneous penetration, hydrophobicity, limited passive diffusion, and high molecular weight (1203 Da). ${ }^{13,14}$ Moreover, physiological factors must be considered, for example, psoriatic lesions may thicken the stratum corneum and epidermis. However, new strategies to improve topical administration of CsA developed such as amphiphilic gels, ${ }^{15}$ microemulsions, ${ }^{16}$ amorphous nanoparticles, ${ }^{17}$ polymeric nanoparticles, ${ }^{18}$ and lipid nanoparticles. ${ }^{9,19,20}$

Polymeric nanoparticles have been extensively utilized as carrier systems to increase the percutaneous absorption of therapeutic agents for transdermal delivery. A number of natural and synthetic polymers have been used as carriers for transdermal delivery. ${ }^{18}$ Eudragit L100 a stimuliresponsive polymer has been used as a carrier in the present study because the $\mathrm{pH}$-dependent dissolution behavior of Eudragit L100 is thought to be very useful in the topical routes because the $\mathrm{pH}$ of the skin is 5.5 while that of the systemic circulation is 7.4. ${ }^{21}$ And anionic acrylic acid-derived polymer Carbopol 934 was selected as a gelling agent due to its excellent gelling properties and rheological behavior. Carbopol 934 has the advantage of greater viscosity at a lower concentration, better thermal stability, and good adhering property. ${ }^{22}$

In the present work, an attempt was made to develop CsA loaded polymeric nanoparticles for topical application. Developed CsA loaded nanoparticles were incorporated into Carbopol 934 based gel which can be used as the final formulation for topical application to increase the permeation and to facilitate the adherence and retention properties of the nanoparticles on the surface of the skin. This delivery system is expected to have better patient compliance, and we have reported the enhancement in the topical delivery of CsA as well. 


\section{MATERIALS AND METHODS}

The selected drug CsA was obtained as a gift sample from Accufine Pharma Pvt. Ltd. (Gujarat, India), Eudragit L100 and Carbopol 934 was purchased from Research Lab Chemical Centre, (Mumbai, India), polyvinyl alcohol, methanol, and acetone belong to Molychem, (Mumbai, India). All other materials or chemicals used were of analytical grade.

\section{Preparation of Cyclosporine A loaded nanoparticles}

CsA loaded nanoparticles were prepared through a modified nanoprecipitation method using the different polymer concentrations as shown in Table $1 .{ }^{23}$ Eudragit L100 and CsA were dissolved in methanol. The mixture formed was added drop-wise into the aqueous solution of polyvinyl alcohol (PVA) as a surfactant and was magnetically stirred at $500 \mathrm{rpm}$ for $180 \mathrm{~min}$ for complete removal of organic solvent. The hardened CsA nanoparticles were recovered by centrifugation at 15000 rpm, washed repeatedly with deionized water, collected, and dried at $40^{\circ} \mathrm{C}$ for $1 \mathrm{~h}$.

\section{Physicochemical characterization of nanoparticles}

\section{Particle size and zeta potential study}

A dynamic light scattering particle size analyzer (Zetasizer Nano S90 Malvern, UK) was used for the measurement of a mean size, zeta potential, and PDI of the prepared nanoparticles. ${ }^{24}$

\section{Encapsulation efficiency and drug loading}

The encapsulation efficiency (\% EE) and drug loading of prepared CsA loaded nanoparticles were determined by dissolving a known quantity of nanoparticles in $10 \mathrm{~mL}$ of methanol under sonication for $1 \mathrm{hr}$. The samples were then filtered through a membrane filter and analyzed at 215 nm using high-performance liquid chromatography (HPLC) (Shimadzu $\mathrm{LC}_{10} \mathrm{AD}$ ). The \% $\mathrm{EE}$ and the loading of CsA into nanoparticles were determined according to the following equations:

$\% \mathrm{EE}=\left[\frac{\text { Actual drug content in nanoparticles }}{\text { Total drug used in formulation }}\right] \times 100$

Table 1: Formulation design for preparation of nanoparticles.

\begin{tabular}{cccc}
\hline $\begin{array}{c}\text { Formulation } \\
\text { code }\end{array}$ & $\begin{array}{c}{ }^{\mathrm{C} C S A} \\
(\mathrm{mg})\end{array}$ & $\begin{array}{c}{ }^{\text {b}} \text { Eudragit L } \\
(\mathrm{mg})\end{array}$ & $\begin{array}{c}\text { cPVA } \\
(\%)\end{array}$ \\
\hline A1 & 100 & 100 & 0.5 \\
A2 & 100 & 200 & 0.5 \\
A3 & 100 & 300 & 0.5 \\
A4 & 100 & 400 & 0.5 \\
A5 & 100 & 500 & 0.5 \\
A6 & 100 & 100 & 1.0 \\
A7 & 100 & 200 & 1.0 \\
A8 & 100 & 300 & 1.0 \\
A9 & 100 & 400 & 1.0 \\
A10 & 100 & 500 & 1.0 \\
A11 & 100 & 100 & 1.5 \\
A12 & 100 & 200 & 1.5 \\
A13 & 100 & 300 & 1.5 \\
A14 & 100 & 400 & 1.5 \\
A15 & 100 & 500 & 1.5 \\
\hline
\end{tabular}

${ }^{\mathrm{a}}$ Cyclosporine; ${ }^{\mathrm{b}}$ Eudragit L100; ${ }^{\mathrm{c}}$ Polyvinyl alcohol
$\%$ Drug Loading $=\left[\frac{\text { (Total amount of drug }- \text { Unincorporated drug amount })}{\text { Amount of nanoparticles recovered }}\right] \times 100$

\section{Morphological analysis}

The surface morphological observations of CsA loaded nanoparticles were examined via a high-resolution scanning electron microscopy (Model JSM 5610 LV SEM, Japan).

\section{Fourier-transform infrared spectroscopy and differential scanning calorimeter analysis}

The Fourier-transform infrared spectroscopy (FTIR) spectra of pure CsA and CsA loaded nanoparticles were obtained by a computerized FTIR spectroscopy (Shimadzu 8400s FTIR spectrometer, Japan) operating in the scanning wave number range of 4000 and $700 \mathrm{~cm}^{-1}$.

\section{Differential scanning calorimeter analysis}

The Differential scanning calorimeter (DSC) thermograms of CsA and CsA loaded Eudragit L100 nanoparticles were recorded by a DSC 60A (Shimadzu, Japan). Samples (10 mg) were sealed into hard-pressed plus perforated crucibles of aluminium and heated from $25^{\circ} \mathrm{C}$ to $300^{\circ} \mathrm{C}$ at a heating rate of $10^{\circ} \mathrm{C} / \mathrm{min}$.

\section{Powder X-ray diffraction analysis}

Powder X-ray diffraction analysis (PXRD) of pure CsA and an optimized batch of CsA loaded nanoparticles were analyzed by Philips PW 1729 $\mathrm{X}$-ray diffractometer. Samples were irradiated with monochromatized $\mathrm{Cu} \mathrm{Ka}$-radiations (operating at $40 \mathrm{kV}, 20 \mathrm{~mA}$ ) and analyzed at a scanning rate of $4^{\circ} \mathrm{C}$ over a $2 \theta$ range of $5-40^{\circ}$.

\section{Preparation of Cyclosporine A loaded nanoparticles incorporated gels}

For the preparation of CsA nanoparticles containing gel, the required quantity of Carbopol 934 was taken in enough quantity of water for 24 h. After that $0.5 \mathrm{~mL}$ of methylparaben $(0.5 \% \mathrm{w} / \mathrm{v})$ added to the hydrated gel and stirred for about $4 \mathrm{~h}$. The gel was neutralized by the addition of triethanolamine drop by drop until a clear transparent gel formed. Then CsA loaded nanoparticles (A9) were incorporated in to gel with mechanical mixing. ${ }^{25}$

\section{Characterization of Cyclosporine A loaded nanoparticulate gel}

The $\mathrm{pH}$ and viscosity of CsA loaded nanoparticulate gel were measured at a ambient temperature by digital $\mathrm{pH}$ meter (Thermo scientific) and Brooke field digital viscometer (DV-II + Pro) respectively. For the measurement of drug content, $100 \mathrm{mg}$ of the CsA nanoparticulate gel was transferred to a $50 \mathrm{ml}$ volumetric flask and was diluted with ethanol. $5 \mathrm{ml}$ of this solution was further diluted to $25 \mathrm{ml}$ with ethanol and resulting samples were analyzed by HPLC at a wavelength of $215 \mathrm{~nm}$ for CsA content.

The spreadability was evaluated by the already reported method. ${ }^{26}$ Briefly, $500 \mathrm{mg}$ of CsA nanoparticulate gel was placed within a circle of $1 \mathrm{~cm}$ diameter pre-marked on the acrylic plate at the center and another plate was concentrically positioned above it. The diameter of the circle in which the CsA nanoparticulate gel was spread was considered as the initial diameter. A weight of $500 \mathrm{~g}$ was then placed on the upper plate for $5 \mathrm{~min}$ and an increase in diameter as a function of applied weight was measured as spreadability of gel.

\section{In vitro release study}

The in vitro release studies of CsA from free drug dispersion, the nanoparticles, and nanoparticulate gel were carried out by dialysis Cassettes (10000 MWCO, Thermo Scientific). A dialysis Cassette 
previously soaked overnight in the diffusion medium and a defined amount of CsA loaded nanoparticles and CsA loaded nanoparticulate gel was accurately inserted using a syringe into dialysis Cassettes. The dialysis Cassettes was suspended in a beaker containing $100 \mathrm{~mL}$ of phosphate buffer $\mathrm{pH} 7.4$ at $37 \pm 0.5^{\circ} \mathrm{C}$. This assembly was kept on a magnetic stirrer at $50 \mathrm{rpm}$. Aliquots of $3 \mathrm{~mL}$ of samples were withdrawn at predetermined time intervals up to $12 \mathrm{hrs}$ and were refilled with a fresh quantity of dissolution medium to maintain sink conditions. The concentration of CsA in the samples was analyzed by HPLC at a wavelength of $215 \mathrm{~nm}$ for CsA content.

\section{In vitro antiproliferative activity}

The potency of CsA nanoparticulate gel was performed using human hyperproliferative keratinocyte cell line (HaCaT). The inhibition of keratinocyte proliferation against homeostatic control of keratinocyte growth and differentiation was measured by in vitro 3-(4,5-dimethylthiazol-2-yl)-2,5-diphenyltetrazolium bromide (MTT) assay. ${ }^{27}$ Briefly, the HaCaT cells were cultured in Dulbecco's modified Eagle's medium supplemented with $10 \%$ fetal bovine serum at $37^{\circ} \mathrm{C}$. The cells were treated with free CsA, blank nanoparticles, and nanoparticles loaded with CsA and incubated for $24 \mathrm{~h}$. After incubation, Coenzyme Q10 was added to wells. Before the MTT test, each well was washed with phosphate-buffer saline (PBS), and 0.5\% MTT 40 was added and then was incubated for $3 \mathrm{hr}$. Finally, Dimethyl sulfoxide was added to dissolve purple formazan and absorption was determined by the absorption in an enzyme-linked immunosorbent assay plate reader at $490 \mathrm{~nm}$ and a reference wavelength at $630 \mathrm{~nm}$. The results were expressed concerning $\%$ growth inhibition.

\section{Dermal pharmacokinetic studies by using tape stripping technique}

The in vitro skin penetration study was performed by tape stripping technique. ${ }^{28-30}$ The skin samples were unclipped from the Franz diffusion cell after $24 \mathrm{~h}$ and the exposed stratum corneum surface of the skin was washed thrice with PBS and was left for air drying. The $19 \mathrm{~mm}$ Scotch cellophane tape (3M, USA) was utilized for this technique. The initial stripped tape was discarded as it contains superficially adhered drugs. To remove the stratum corneum layer, 15 strips were detached in a manner that the entire area of the tape was utilized. The tape samples were digested with a mixture of ethyl acetate and tetrahydrofuran (50:50). The tape, as well as skin samples, was soaked in methanol overnight to allow the extraction of the drug from stratum corneum and deeper layers respectively and further kept in the bath sonicator for $1 \mathrm{hr}$ for the complete drug extraction. Then the extracted samples were analyzed by the HPLC method. The quantity present in the receptor compartment was the index of transdermal delivery (systemic absorption), whereas in the tape and skin extract was the index of topical drug delivery.

\section{RESULTS}

\section{Physicochemical characterization of nanoparticles}

\section{Particle size and zeta potential study}

The average particle size and zeta potential values of CsA loaded nanoparticles were presented in Table 2.

\section{Encapsulation efficiency and drug loading}

The finding showed that the entrapment efficiency as well as drug loading was higher at $400 \mathrm{mg}$ of polymer with a $1 \%$ concentration of surfactants. Formulation A9 had the highest entrapment efficiency and the maximum percentage of drug loading presented in Table 3.

\section{Morphological analysis}

The surface morphological study of CsA loaded nanoparticles were done with scanning electron microscopy (Model JSM 5610 LV SEM, Japan). The Scanning electron microscopy of CsA loaded nanoparticles showed relatively smooth surface nanoparticles distributed uniformly and have spherical shapes.

\section{Fourier-transform infrared spectroscopy}

The FTIR study was carried out to check the compatibility between CsA and polymer. The comparative FTIR peaks of pure CsA and CsA loaded nanoparticles represented in Figure 1.

\begin{tabular}{ccc|}
$\begin{array}{c}\text { Table 2: Mean particle size and zeta potential of } \\
\begin{array}{c}\text { Formulation } \\
\text { code }\end{array}\end{array}$ & $\begin{array}{c}\text { Particle size } \\
(\mathrm{nm})\end{array}$ & $\begin{array}{c}\text { Zeta potential } \\
(\mathrm{mV})\end{array}$ \\
\hline A1 & $144 \pm 4.22$ & $0.69 \pm 0.56$ \\
A2 & $132 \pm 0.96$ & $0.71 \pm 0.74$ \\
A3 & $126 \pm 2.84$ & $0.73 \pm 0.46$ \\
A4 & $114 \pm 2.01$ & $0.69 \pm 0.72$ \\
A5 & $106 \pm 2.08$ & $0.70 \pm 0.11$ \\
A6 & $87 \pm 1.32$ & $0.72 \pm 0.12$ \\
A7 & $71 \pm 1.56$ & $0.70 \pm 0.46$ \\
A8 & $66 \pm 1.28$ & $0.65 \pm 0.27$ \\
A9 & $58 \pm 0.22$ & $0.59 \pm 0.13$ \\
A10 & $63 \pm 1.42$ & $0.62 \pm 0.22$ \\
A11 & $96 \pm 1.32$ & $0.68 \pm 0.24$ \\
A12 & $105 \pm 0.42$ & $0.77 \pm 0.21$ \\
A13 & $126 \pm 2.32$ & $0.91 \pm 0.11$ \\
A14 & $142 \pm 2.14$ & $0.94 \pm 0.12$ \\
A15 & $146 \pm 3.28$ & $0.96 \pm 0.32$ \\
\hline
\end{tabular}

Data are represented as Mean $\pm \operatorname{SD}(n=3)$

Table 3: Entrapment efficiency and drug loading of nanoparticles.

\begin{tabular}{ccc}
$\begin{array}{c}\text { Formulation } \\
\text { code }\end{array}$ & $\begin{array}{c}\text { Entrapment efficiency } \\
(\%)\end{array}$ & $\begin{array}{c}\text { Drug loading } \\
(\%)\end{array}$ \\
\hline A1 & $35.19 \pm 1.3$ & $48.02 \pm 0.04$ \\
A2 & $36.24 \pm 1.6$ & $60.22 \pm 1.11$ \\
A3 & $37.12 \pm 1.4$ & $67.78 \pm 2.06$ \\
A4 & $35.48 \pm 1.2$ & $60.02 \pm 1.02$ \\
A5 & $36.14 \pm 2.0$ & $66.62 \pm 1.03$ \\
A6 & $38.26 \pm 1.2$ & $64.24 \pm 1.12$ \\
A7 & $37.18 \pm 1.4$ & $58.89 \pm 1.14$ \\
A8 & $39.08 \pm 1.4$ & $61.13 \pm 0.26$ \\
A9 & $41.15 \pm 1.3$ & $69.17 \pm 1.34$ \\
A10 & $38.28 \pm 1.2$ & $56.45 \pm 1.15$ \\
A11 & $32.66 \pm 1.3$ & $61.18 \pm 2.09$ \\
A12 & $30.22 \pm 1.1$ & $65.48 \pm 1.84$ \\
A13 & $29.24 \pm 1.2$ & $57.12 \pm 1.48$ \\
A14 & $27.18 \pm 1.2$ & $62.87 \pm 1.13$ \\
A15 & $26.50 \pm 1.0$ & $56.42 \pm 1.21$ \\
\hline
\end{tabular}

Values are reported as Mean $\pm \mathrm{SD}(n=3)$ 


\section{Differential scanning calorimeter (DSC) analysis}

DSC study of pure CsA and CsA loaded nanoparticles was performed to investigate the crystalline or amorphous nature of formulation as well as to find out the interaction between drug, polymer, and other excipients.

\section{Powder X-ray diffraction analysis}

The PXRD technique was employed to measure the physical state of the encapsulated drug which represented in Figure 2.

\section{Characterization of Cyclosporine A loaded nanoparticulate gel}

The $\mathrm{pH}$ of CsA loaded nanoparticulate gel was found to be 6.8 and the drug content was found to be $97.4 \%$. The spreadability of CsA loaded nanoparticulate gel were $41.15 \pm 1.3 \mathrm{~cm}$.

\section{In vitro release study}

The data of percentage drug release formulation was shown in Figure 3. The amount of CsA from free drug dispersion demonstrated $96.99 \%$ release in $1 \mathrm{hr}$, while the release behavior of CsA from nanoparticles dispersion and the nanoparticulate gel was controlled over a time period of $12 \mathrm{~h}$. It was observed that approximately $75.45 \%$ of CsA release in case of CsA nanoparticles dispersion, while $69.23 \%$ of CsA release after $12 \mathrm{~h}$ in the case of CsA nanoparticulate gel.

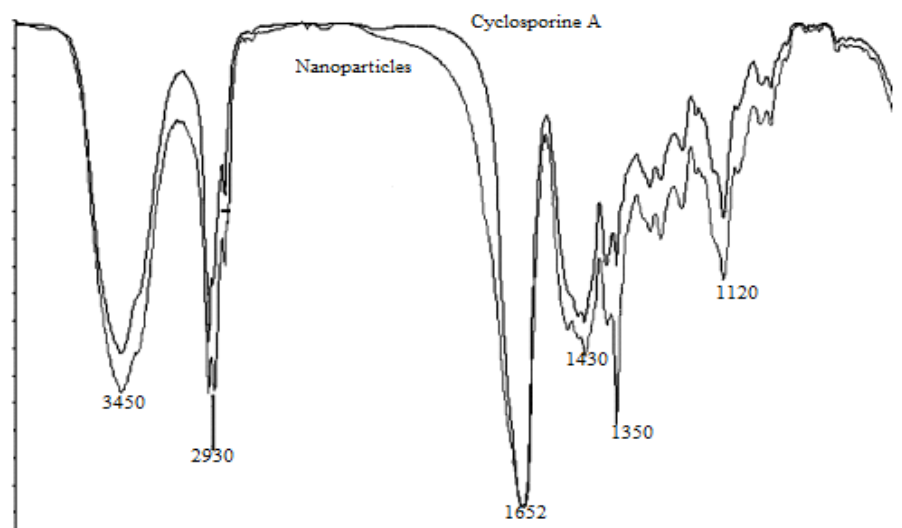

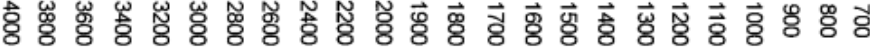$$
\mathrm{Cm}^{-1}
$$

Figure 1: Comparative FTIR spectra of pure CsA and CsA loaded nanoparticles.
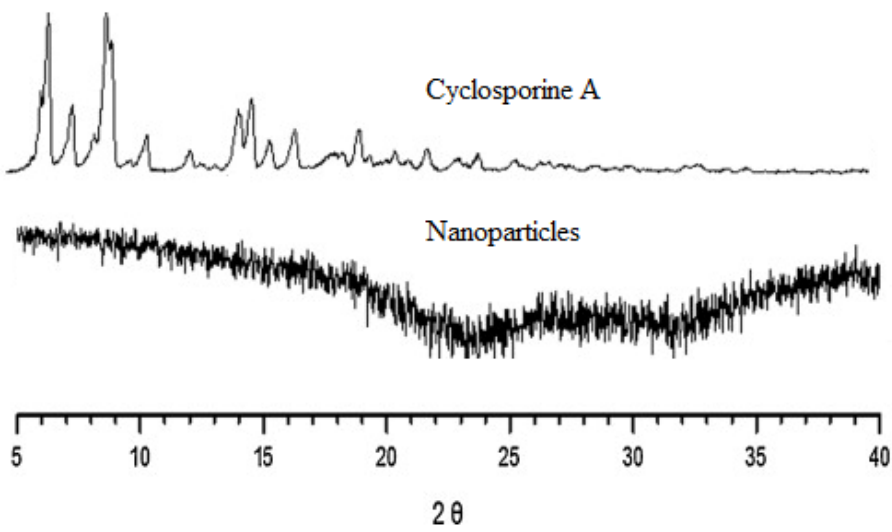

Figure 2: X-ray diffraction pattern of CsA and CsA loaded nanoparticles (A9).

\section{In vitro antiproliferative activity}

These cell lines efficiently predict the uptake of formulations by the cells. The results are shown in Figure 4(a), which explains the percent viability against the dose.

\section{Dermal pharmacokinetic studies by using tape stripping technique}

The dermal pharmacokinetic investigations presented in Figure 4 (b) clearly showed that all the formulations were uniformly distributed in stratum corneum and viable layers.

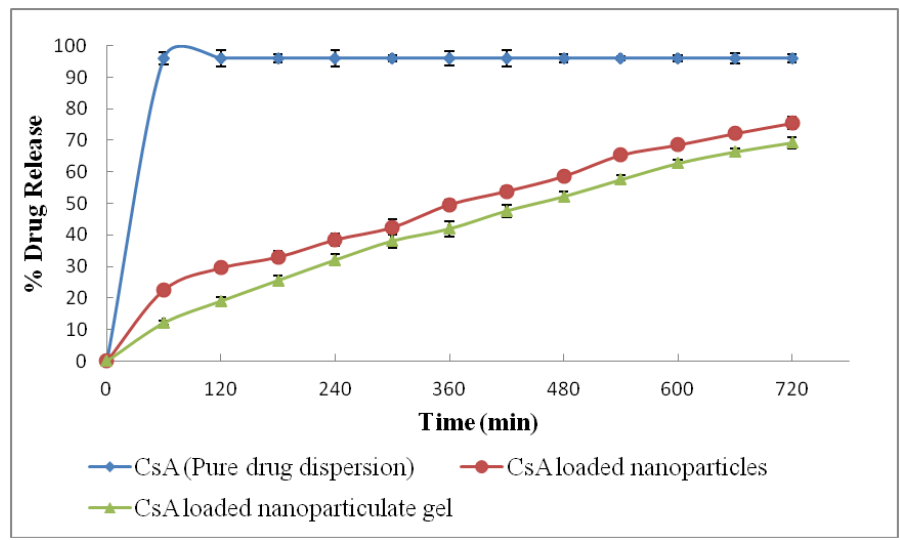

Figure 3: In vitro drug release profile of CsA from free drug dispersion, CsA nanoparticles and CsA nanoparticulate gel.

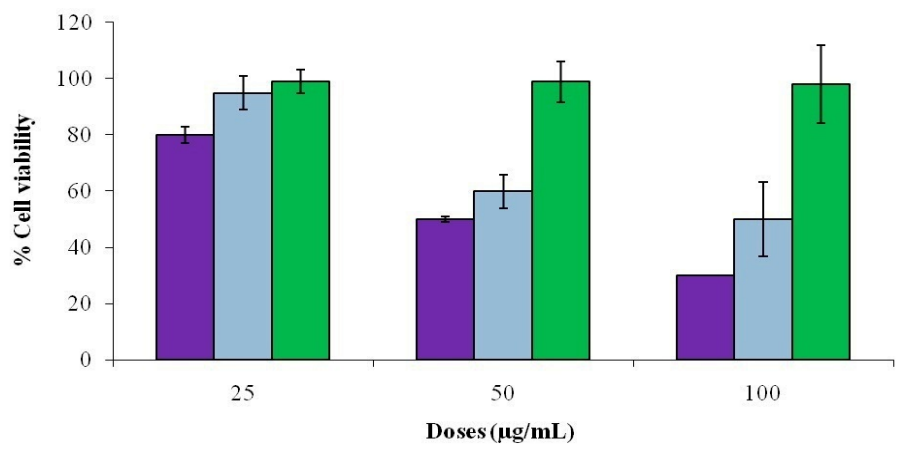

$\square$ Nanoparticles loaded with Cyclosporine $\square$ Free Cyclosporine A $\square$ Blank nanoparticles

(a)

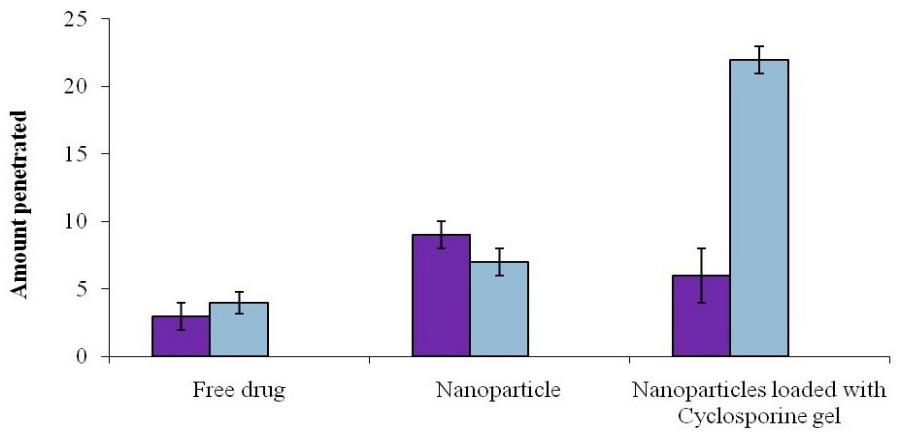

口Stratum corneum $\quad$ QViable layers

Figure 4: (a) Comparative evaluation of $\%$ cell viability of free drug, blank nanoparticles, and CsA loaded nanoparticles (b) Quantification of CsA in different skin layers in case of free drug, nanoparticle and CsA loaded nanoparticulate gel (Values are expressed as mean $\pm S D, n=3$ ). 


\section{DISCUSSION}

Eudragit L 100 is a positively charged polymer that imparts cationic nature to CsA loaded nanoparticles. ${ }^{23}$ It was observed that the higher the value of zeta potential lesser the aggregation of the particle, attributable to electric repulsion and consequently greater the stability of CsA loaded nanoparticles. It was experiential that positive charge shows on the surface of CsA loaded nanoparticles can be recognized to the existence of the quaternary ammonium groups of Eudragit L100 polymer. $^{23}$

The basic peaks in FTIR study was retained in the pure CsA and CsA loaded nanoparticles (A9). Presence of $\mathrm{C}-\mathrm{H}$ stretching in the region of 2850-3000 $\mathrm{cm}^{-1}$ and $\mathrm{CH}_{3}$ bending at $1350 \mathrm{~cm}^{-1}$, the emergence of the strong band found in between $1600-1720 \mathrm{~cm}^{-1}$ presents the existence of carbonyl group mainly because of $\mathrm{C}=\mathrm{O}$ stretching, $\mathrm{COO}$ stretching at $1000-1300 \mathrm{~cm}^{-1}$ established the presence of CsA in CsA loaded nanoparticles (A9). The optimized formulation A9 showed an indistinguishable spectrum respecting the spectrum of the pure CsA indicating no chemical interaction or changes between the CsA and Eudragit L100 during the preparation of nanoparticles.

DSC analysis showed a sharp endothermic peak at $134^{\circ} \mathrm{C}$ for pure CsA equivalents to it's melting point. While in CsA loaded nanoparticles melting endotherm was observed at $136^{\circ} \mathrm{C}$ with a sharp appearance, this slight reduction in temperature may be because of reductions of the crystallinity nature of CsA in the formulation of nanoparticles.

PXRD stated that the intensity of the peaks for the pure CsA was sharp but for CsA loaded nanoparticles (A9), the intensities of the peaks decreased as a result, the reduction of the crystallinity of the CsA.

CsA loaded nanoparticles were uniformly distributed within the gel which exhibited non-newtonian flow i.e. thixotropic (pseudoplastic) rheology as evidenced by a decrease in viscosity with an increase in shear rate.

Spreadability plays an important role in patient compliance and helps in the uniform appliance of gel to the skin. Topical gels prepared with the low concentration of Carbopol 934 belonged to the fluid gel category, having more spreadability values and formulations prepared with the higher concentration of Carbopol 934 belonged to a stiff and semi-stiff category. The results indicated that the formulation can be applied easily without being the runoff. ${ }^{31}$ This assures that the formulation maintains a good wet contact time when applied to the site of application.

Mathematical modeling was done using DD Solver, and it was suggested that free CsA dispersion follow zero-order kinetics $\left(R^{2}=0.9617\right)$, while CsA nanoparticles dispersion $\left(R^{2}=0.9708\right)$ and CsA nanoparticulate gel $\left(R^{2}=0.9897\right)$ demonstrated Higuchi type matrix release behavior. It is suggested that the diffusion-dependent release behavior of CsA via erosion of the solid matrix of polymers or gel may be the primary mechanism for the release of the drug. Carbopol 934 gel matrix presented an additional barrier, so further retarded the drug release from CsA nanoparticulate gel in comparison to CsA nanoparticles dispersion. Furthermore, higher viscosity of gel matrix decreases the release rate of CsA as a result of lesser penetration of water and thus, hydration of gel matrix.

In vitro antiproliferative study proved that $50 \%$ of the cells were inhibited by CsA loaded nanoparticles when a dose of $50 \mu \mathrm{g} / \mathrm{mL}$ was given and cell viability diminished gradually as the dose was raised. On the other hand, free CsA was not able to inhibit $50 \%$ of the growth, when the highest dose of $100 \mu \mathrm{g} / \mathrm{ml}$ was given, thereby proving the fact that nanoparticles had a better effect on cell growth as compared to the free drug which was in turn due to higher uptake by the cells. ${ }^{32}$ It was also observed that cell viability was not affected with blank nanoparticles, which proved there was no effect of surfactant concentration and surfactant type on cell viability causing no cell death.
In dermal pharmacokinetic study for the CsA loaded nanoparticulate gel, the amount reaching the viable layers is slightly higher than the stratum corneum. Psoriatic skin is rough, hard, and flaky due to it is hard to penetrate. The presence of gel hydrates the skin which helps in the higher permeation of CsA loaded nanoparticulate gel. The nanoparticle on the other hand acted more efficiently on the stratum corneum as they face difficulty penetrating into deeper layers of the skin. In the case of the free drug the amount of drug in the stratum corneum, as well as the epidermis and a viable layer, was less, therefore explaining their minimal penetration into the skin. ${ }^{29}$

\section{CONCLUSION}

In the present investigation, CsA nanoparticles were successfully formulated using a modified nanoprecipitation technique, and subsequently converted into the gel using Carbopol 934 as a gelling agent. The developed CsA nanoparticulate gel formulation improved dermal delivery of CsA and exhibits enhanced in vitro anti-psoriatic efficacy. The in vitro antiproliferative activity proved that nanoparticles had a superior effect on cell growth as compared to the free CsA. Dermal pharmacokinetic study demonstrated that for CsA nanoparticulate gel the amount of CsA reaching the viable layer is slightly high as compared to the stratum corneum. These hopeful in vitro results can be transformed into the probably marketed product after successful in vivo study as well as clinical trials in humans.

\section{ACKNOWLEDGEMENT}

The authors acknowledge the Nootan Pharmacy College, Sankalchand Patel University, Visnagar, Gujarat, India for providing necessary infrastructure facilities for carry out this investigation.

\section{CONFLICT OF INTEREST}

The authors declare no conflicts of interest.

\section{ABBREVIATIONS}

CsA: Cyclosporine A; T-cell: T lymphocyte; Da: Dalton; PDI: Poly Dispersity Index.

\section{REFERENCES}

1. Parisi R, Symmons DP, Griffiths CE, Ashcroft DM. Global epidemiology of psoriasis: A systematic review of incidence and prevalence. J Invest Dermatol. 2013;133(2):377-85

2. Cameron JB, Voohees AS. History of Psoriasis. Advances in Psoriasis. London: Springer. 2014;1-7.

3. Perera GK, DiMeglio P, Nestle FO. Psoriasis. Annu Rev Pathol. 2012;7:385-22.

4. Tzu J, Mamelak AJ, Sauder DN. Current advancements in the treatment of psoriasis: Immunobiologic agents. Clinical and Applied Immunology Reviews. 2006;6(2):99-130.

5. Chapman BP, Moynihan J. The brain-skin connection: Role of psychosocial factors and neuropeptides in psoriasis. Expert Rev Clin Immunol. 2009;5(6):623

6. Feldman SR, Malakouti M, Koo JY. Social impact of the burden of psoriasis: Effects on patients and practice. Dermatol Online J. 2014;20(8):13030.

7. Raychaudhuri SK, Maverakis E, Raychaudhuri SP. Diagnosis and classification of psoriasis. Autoimmun Rev. 2014;13(4-5):490-5.

8. Gudjonsson J, Johnston A, Sigmundsdottir $H$, Valdimarsson $H$. Immunopathogenic mechanisms in psoriasis. Clin Exp Immunol. 2004;135(1):1 8.

9. Guada M, Sebastian V, Irusta S, Feijoo E, Dios-Vieitez MC, Blanco-Prieto MJ. Lipid nanoparticles for cyclosporine a administration: Development, characterization and in vitro evaluation of their immunosuppression activity. Int J Nanomedicine 2015;10:6541-53.

10. Gupta NV, Kowshik K, Kanna S. A review on etiology and challenges associated with various therapies for the treatment of psoriasis. Int J Pharm Sci and Res. 2019;10(10):4409-19.

11. Griffiths $C$, Dubertret $L$, Ellis C, Finlay A, Finzi A, Ho V, et al. Ciclosporin in psoriasis clinical practice: An international consensus statement. $\mathrm{Br} \mathrm{J}$ Dermatol. 
2004;150(Suppl 67):11-23.

12. Fellstrom B. Cyclosporine nephrotoxicity. Transplant Proc. 2004;36(Supp 2):220S-3S

13. Colombo MD, Cassano N, Bellia G, Vena GA. Cyclosporine regimens in plaque psoriasis: An overview with special emphasis on dose, duration and old and new treatment approaches. Sci World J. 2013;2013:805705.

14. Bos JD, Meinardi MM. The 500 Dalton rule for the skin penetration of chemical compounds and drugs. Exp Dermato. 2000;9(3):165-9.

15. Prasad V, Kumar N, Mishra PR. Amphiphilic gels as a potential carrier for topical drug delivery. Drug Deliv. 2007;14(2):75-85.

16. Liu SLH, WangY, LangY, Yao H, DongY. Bicontinuous Cyclosporin a Loaded WaterAOT/Tween 85-Isopropylmyristate Microemulsion: Structural Characterization and Dermal Pharmacokinetics in vivo. J Pharm Sci. 2009;98(3):1167-76.

17. Romero GB, Arntjen A, Keck CM, Muller RH. Amorphous cyclosporin a nanoparticles for enhanced dermal bioavailability. Int J Pharm. 2016;498(12):217-24.

18. Qindeel M, Ahmed N, Sabir F, Khan S, Ur-Rehman A. Development of novel pHsensitive nanoparticles loaded hydrogel for transdermal drug delivery. Drug Dev Ind Pharm. 2019;45(4):629-41.

19. Alvarez-Trabado J, DieboldY, Sanchez A. Designing lipid nanoparticles for topical ocular drug delivery. Int J Pharm. 2017;532(1):204-17.

20. Arora R, Katiyar SS, Kushwah V, Jain S. Solid lipid nanoparticles and nanostructured lipid carrier-based nanotherapeutics in treatment of psoriasis: A comparative study. Expert Opin Drug Deliv. 2014;14(2):165-77.

21. Schmid-Wendtner $\mathrm{MH}$, Korting HC. The pH of the skin surface and its impact on the barrier function. Skin Pharmacol Physiol. 2006;19(6):296-302

22. Islam MT, Rodriguez-Hornedo N, Ciotti S, Ackermann C. Rheological characterization of topical carbomer gels neutralized to different $\mathrm{pH}$. Pharm
Res. 2004;21(7):1192-9.

23. Salatin S, Barar J, Barzegar-Jalali M, Adibkia K, Kiafar $F$ Jelvehgari $M$. Development of a nanoprecipitation method for the entrapment of a very water soluble drug into Eudragit RL nanoparticles. Res Pharm Sci. 2017;12(1):1-14.

24. Betancourt T, Brown B, Brannon-Peppas L. Doxorubicin-loaded PLGA nanoparticles by nanoprecipitation: Preparation, characterization and in vitro evaluation. Nanomedicine. 2007;2(2):219-32.

25. Prusty AK, Parida P. Development and Evaluation of Gel Incorporated with Biogenically Synthesised Silver Nanoparticles. J Appl Biopharm Pharmacokinet. 2015;3:1-6.

26. Shah KA, Date AA, Joshi MD, Patravale VB. Solid lipid nanoparticles (SLN) of tretinoin: Potential in topical delivery. Int J Pharm. 2007;345(1-2):163-71.

27. Lau WM, Ng KW, White AW, Heard CM. Therapeutic and cytotoxic effects of the novel antipsoriasis codrug, naproxyl-dithranol, on HaCaT Cells. Mol Pharm. $2011 ; 8(6): 2398-407$.

28. N'Dri-Stempfer B, Navidi WC, Guy RH, Bunge AL. Improved bioequivalence assessment of topical dermatological drug products using dermatopharmacokinetics. Pharm Res. 2009:26(2):316-28.

29. NavidiW, Hutchinson A, N'Dri-Stempfer B, Bunge A. Determining bioequivalence of topical dermatological drug products by tape-stripping. J Pharmacokinet Pharmacodyn. 2008;35(3):337-48.

30. Wu X, Griffin P, Price GJ, Guy RH. Preparation and in vitro evaluation of topical formulations based on polystyrene-poly-2-hydroxyl methacrylate nanoparticles. Mol Pharm. 2009;6(5):1449-56.

31. Naga SKVV, Maheshwari PV, Navya M, Chandra RS, Shivakumar HG, Gowda DV Calcipotriol Delivery into the Skin as Emulgel for Effective Permeation. Saudi Pharm J. 2014;22(6):591-9

32. Kumar V, Sharma N, Maitra SS. In vitro and in vivo toxicity assessment of nanoparticles. Int Nano Lett. 2017;7(4):243-56

Article History: Submission Date : 19-12-2020; Revised Date : 20-01-2021; Acceptance Date : 26-02-2021

Cite this article: Prajapati R, Patel J, Patel A. Nanoparticles Containing Gel Formulation for the Treatment of Psoriasis. Int. J. Pharm. Investigation. 2021;11(1): 76-81. 